\title{
An illustrated guide of ferns and lycophytes from Carambeí, PR, Brazil
}

\author{
Cássio Michelon ${ }^{1}$, Frederico Fregolente Faracco Mazziero ${ }^{1,2}$, Bianca Kalinowski Canestraro ${ }^{1}$ \\ \& Mathias Erich Engels ${ }^{1}$
}

\begin{abstract}
We present a list of species of ferns and lycophytes from Carambeí, a municipality located in Paraná state, southern Brazil. This area, locally known as "Campos Gerais", presents an intricate mosaic of forests and savannah with several canyons and humid valleys that harbors a rich and unique vegetation in Southern Brazil. In total, we found 129 species (119 ferns and 10 lycophytes), distributed in 18 families and 59 genera, and 20\% (26 spp.) of which are endemic to Brazil. The most expressive families were Polypodiaceae (19 spp.), Pteridaceae (18 spp.) and Thelypteridaceae (15 spp.). The most representative genera were Asplenium (8 spp. - 6.2\%) and Amauropelta (8 spp - 6.2\%). Terrestrial herbs, epiphytes and lithophytes are the most common guild of life forms, successively. The richest environments were woody forest ( $61 \%$ of the species) and herbaceous-shrubby (12\%). Arachniodes denticulata, Phlegmariurus flexibilis and P. heterocarpon are the second record in the "Campos Gerais" region. We also provide photographic plates with diagnostic characters for most of the species.

Key words: Campos Gerais, diversity, floristic, pteridophytes, species richness.

\section{Resumo}

É apresentada uma listagem de espécies de samambaias e licófitas para o município de Carambeí, localizado no estado do Paraná, Sul do Brasil. Essa área, localmente conhecida como Campos Gerais, apresenta um complexo mosaico de florestas e Cerrado com diversos canyons e vales úmidos e comporta uma vegetação rica e singular no Sul do Brasil. No total, foram registradas 129 espécies (119 samambaias e 10 licófitas), distribuídas em 18 famílias e 59 gêneros, sendo 20\% (26 spp.) endêmicas do Brasil. As famílias mais expressivas são Polypodiaceae (19 spp.), Pteridaceae (18 spp.) e Thelypteridaceae (15 spp.). Os gêneros mais representativos foram Asplenium (8 spp. - 6,2\%) e Amauropelta (8 spp. - 6,2\%). As guildas de formas de vida mais comuns são ervas terrícolas, epífitas e rupícolas, sucessivamente. Os ambientes mais ricos foram os florestais (61\% das espécies) e os herbáceo-arbustivos (12\%). Arachniodes denticulata, Phlegmariurus flexibilis e $P$. heterocarpon foram registradas pela segunda vez para a região dos Campos Gerais. São fornecidas pranchas fotográficas com caracteres diagnósticos para a maioria das espécies.

Palavras-chave: Campos Gerais, diversidade, florística, pteridófitas, riqueza de espécies.
\end{abstract}

\section{Introduction}

Ferns and lycophytes are an important component of the vascular flora in tropical forests, representing $10 \%$ to $14 \%$ of the diversity in these environments, often being the dominant groups in the understory (Gentry 1990; Costa 2004; Moran 2008). In Brazil, these groups are represented by 1,318 species, $503(38 \%)$ of which are endemic (Prado et al. 2015). The Atlantic Forest is the most diverse domain in Brazil harboring 883 species and the Ombrophilous Forest is the type of vegetation with greatest diversity with $90 \%$ of the species in this domain (Prado et al. 2015). This diversity is reflected in several studies carried out in these areas, such as, Mynssen \& Windisch (2004) for Rio de Janeiro state, Salino \& Almeida (2008) and Mazziero et al. (2015) for São Paulo state and Matos et al. (2010) for Bahia state.

In the state of Paraná, the areas of Atlantic Rain Forests in the coastal region are responsible for a high richness of ferns and lycophytes (Prado

\footnotetext{
${ }^{1}$ Universidade Federal do Paraná, Depto. Botânica, C.P. 19031, 81531-980, Curitiba, PR, Brazil.

${ }^{2}$ Author for correspondence: fredericobio2@gmail.com
} 
et al. 2015), which is strongly related with the great diversity of available environments in mountainous areas (Moran 1995). In contrast, in the inland of Paraná, the relief of the "Campos Gerais" region is not mountainous, but it presents a unique arrange of geomorphologic characteristics (Maack 1981). Recent studies carried out in these areas (Schwartsburd and Labiak 2007; Michelon \& Labiak 2013) have shown the importance of inland regions to the diversity of ferns and lycophytes as well. Nevertheless, few studies have been performed in the inland portion of Paraná, for example Sakagami (2006), Schwartsburd \& Labiak (2007), Michelon \& Labiak (2013), Lautert et al. (2015) and, new species of ferns from this region have been recently described (Schwartsburd et al. 2007; Schwartsburd \& Labiak 2008; Christenhusz et al. 2009). Therefore, floristics studies and plant collections are still crucial considering the distinct mosaic and the high diversity of plants in these areas. It is important to mention that most of these studied areas are conservation units with minor human impacts. Recent data show that there is left no more than $12 \%$ of Atlantic Forest remnants in Paraná state, and with very few inland forested areas (Fundação SOS Mata Atlântica \& INPE 2016). The inland portions of the state suffered a severe deforestation due to agriculture and livestock activities.

This study aims to contribute to the knowledge of the diversity of these groups in the "Campos Gerais" region, providing a list of species, as well as data about geographic distribution, phytophysiognomy and guild of life forms. We also provide photographic records with diagnosis characters for most of the species.

\section{Materials and Methods}

Study area

Carambeí is located in Paraná state, southern Brazil (2457'04'S, 5006'37' W). Its territory is about $650 \mathrm{~km}^{2}$ occupying the "Campos Gerais" region in the Paraná's First and Second Plateaus (Maack 1981; Cordeiro Santos et al. 2009; Labiak 2014a). The altitude ranges from $780 \mathrm{~m}$ a.s.1., in the proximities of the Tibagi river, to $1,100 \mathrm{~m}$ a.s.1., at the top of the Devonian Steep.

In the Paraná's First Plateau predominates the Araucaria forest and small patches of intensively disturbed grasslands. In the Second Plateau, associated with capon forests, the grasslands dominate the landscape. Also, Cerrado relicts (Brazilian savannah) can be found in the São João river canyon (Maack 1981).
Field work

Collections were performed during March 2013. All possible habitats were explored. In this expedition, the specimens were collected and herborized following the usual methods for the studied groups (Fidalgo \& Bononi 1984) and were deposited in the UPCB herbarium.

\section{Taxonomic treatment}

Classification system followed PPG I (2016). Species' authors and abbreviations followed IPNI (2017). Identifications were made by comparison with collections at UPCB, specialists and specific literature. The guilds of life forms were based on Paciencia (2008).

\section{Geographical distribution}

Geographical distribution data were obtained from Prado et al. (2015), floras and review papers. The geographic patterns adopted were: Pantropic, Neotropic, South America, Brazil and Southern/ Southeastern Brazil.

\section{Vegetation}

Regarding the environments in which the species were found, we considered three situations:

Aquatic vegetation:

Alagados reservoir (Fig. 1a): floating vegetation in the Pitanguí hydroelectric power plant reservoir.

Woody vegetation: arboreal vegetation, mid to large size, primitive (primary forests) or secondary (disturbed forests and capoeirões), divided into:

Secondary forests (Fig. 1b,c): secondary vegetation from initial to medium regeneration stage.

Gallery forests (Fig. 1d,e): forests growing on geological fissures, composed by primary vegetation, usually with rocky streams inside.

Riparian forests (Figs. 1f; 2a): forests that grow along the major rivers in the area (Jotuva, Pitanguí, São João, Tamanduá and Tibagi rivers).

Riparian slabstone environments (Fig. 2b): midsize vegetation growing alongside stony rivers amid grasslands areas, usually in the expansions of the rock-forming slabstone.

Swamp areas: midsize woody vegetation in areas of poorly drained soil, usually amid grasslands, however, without undergoing periodic flooding.

Herbaceous and shrubby vegetation: small vegetation, herbaceous and/or shrubby, primitive (grasslands) or secondary (capoeirinhas), divided into: 

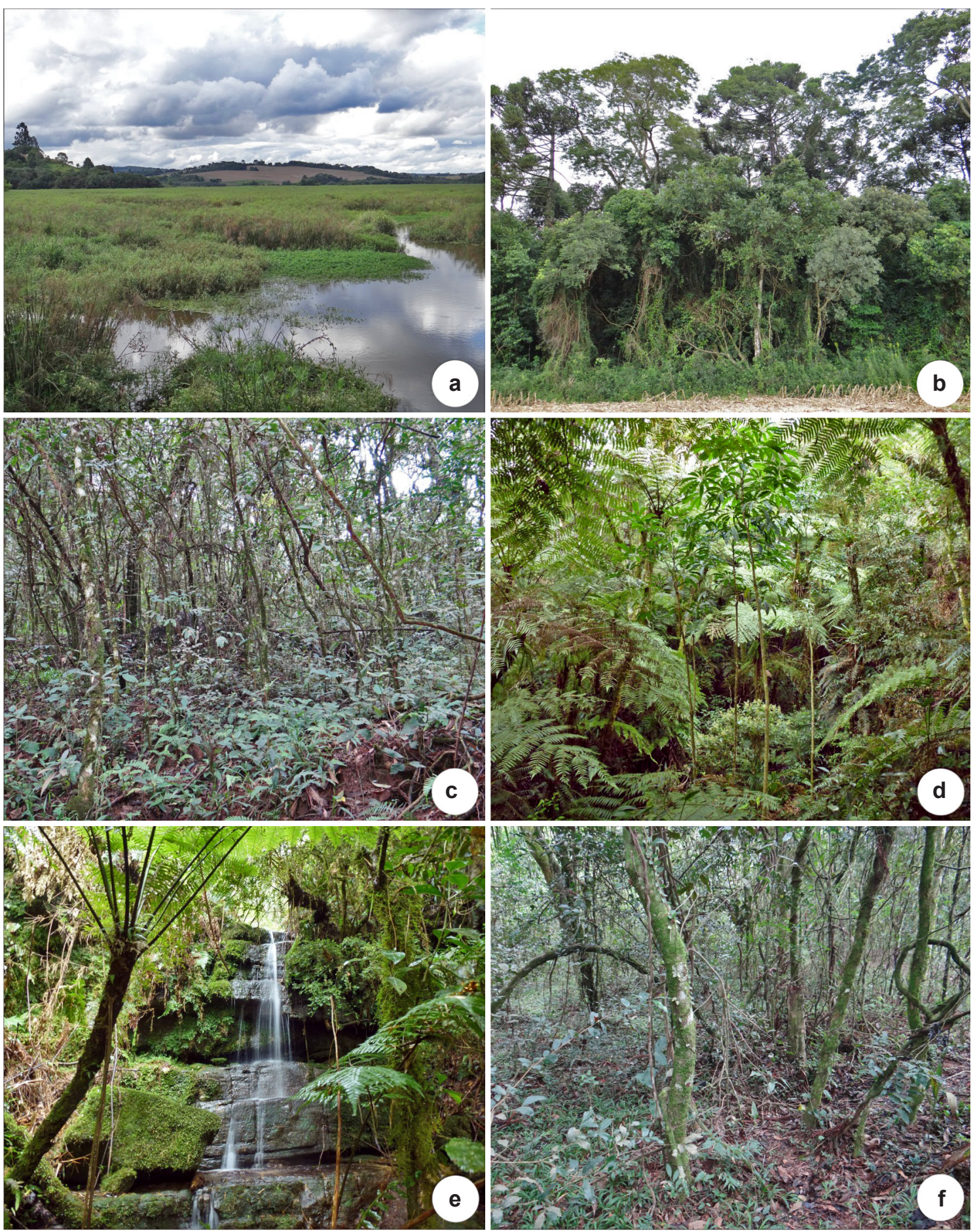

Figure 1 - Aquatic and woody vegetation - a. Alagados' reservoir; b. secondary forest border; c. capon forest understory; d,e. gallery forest understory; f. riparian forest understory. 

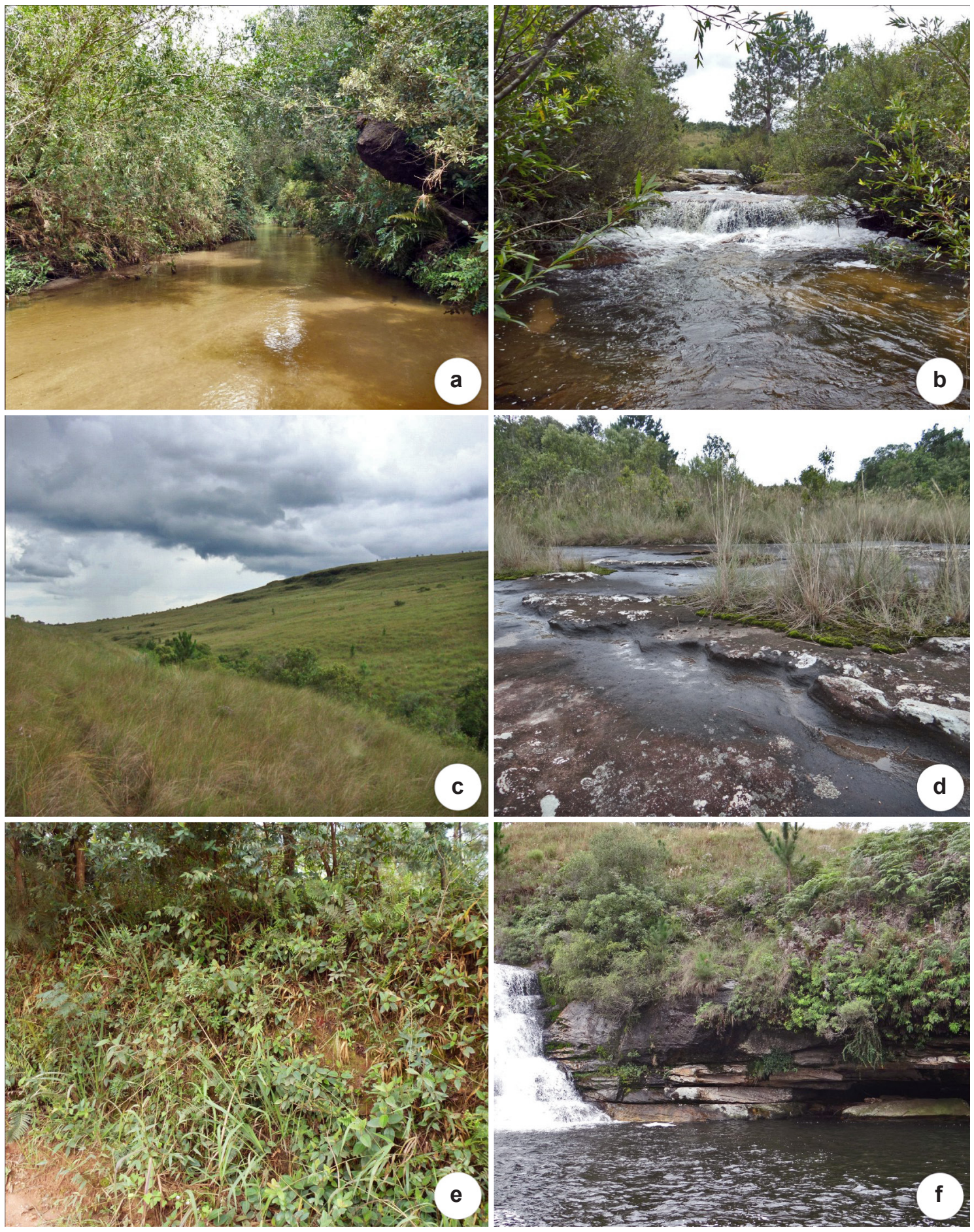

Figure 2 - Woody and herbaceous-shrubby vegetation - a. riparian forest; b. riparian slabstone environment; c. dry grasslands; d. litholic grasslands; e. human disturbed environment; f. waterfall amidst grasslands. 
Dry grasslands (Fig. 2c): including areas of campo limpo, where the herbaceous vegetation predominates amongst rare woody species, and campo sujo, where the shrubby and woody species are commonly found. Remnants of Cerrado (Brazilian Savannah) are also included in this category.

Litholic grasslands (Fig. 2d): herbaceous and shrubby vegetation occurring in areas of rocky fields with sandstone outcrops.

Human disturbed environment (Fig. 2e): characterized by intensively modified and mostly herbaceous vegetation, such as urbanized areas and roadsides slopes.

Wet grasslands: mainly herbaceous vegetation in areas of poor water drainage (Fig. 2f).

\section{Results}

In the present work we found 129 species (119 ferns and 10 lycophytes), distributed in 18 families and 59 genera (Tab. 1 - see supplementary material at $<$ https://doi.org/10.6084/ m9.figshare.6142595.v1 >). The richest families were Polypodiaceae (19 species - 14.7\%), Pteridaceae (18 spp. - 13.9\%), Thelypteridaceae (15 spp. - 11.6\%), Dryopteridaceae (13 spp. - 10\%) and Hymenophyllaceae (12 spp. - 9.3\%), which together comprise almost $60 \%$ of the total richness. The most expressive genera were Asplenium (8 species - 6.2\%), Amauropelta (8 species - 6.2\%) and, Doryopteris, Hymenophyllum and Pecluma (6 species - $4.6 \%$ each) (Tab. 1).

Table 1 - List of ferns and lycophytes recorded in Carambeí municipality, southern Brazil. The life forms are represented by: $\mathrm{T}=$ terrestrial herb, $\mathrm{E}=$ epiphyte, $\mathrm{L}=$ lithophyte $\mathrm{C}=$ climbing, $\mathrm{A}=$ terrestrial arborescent, $\mathrm{H}=$ hemiepiphyte, $\mathrm{Q}=$ aquatic; vegetation: $\mathrm{SF}=$ secondary forest, $\mathrm{DE}=$ human disturbed environment, $\mathrm{LG}=$ litholic grassland, $\mathrm{GF}$ $=$ gallery forest, $\mathrm{RF}=$ riparian forest, $\mathrm{SW}=$ swamp areas, $\mathrm{RS}=$ riparian slabstone environment, $\mathrm{DG}=$ dry grassland, $\mathrm{WG}=$ wet grassland, $\mathrm{RE}=$ Alagados reservoir; geographical distribution: $\mathrm{NEO}=$ Neotropical, $\mathrm{SAM}=$ South American, PAN $=$ Pantropical, BRA $=$ Brazilian, SSE $=$ southern/southeastern Brazilian. Voucher (voucher material at UPCB herbarium: \# - Michelon, C. collector number, * Canestraro B.K. collector number, ! Engels, M.E. collector number).

\begin{tabular}{|c|c|c|c|c|}
\hline $\begin{array}{l}\left.\text { FAMILY ( }{ }^{0} \text { of species }\right) \\
\text { Species }\end{array}$ & Life form & Vegetation & Distribution & Voucher \\
\hline \multicolumn{5}{|l|}{ ANEMIACEAE (3) } \\
\hline Anemia phyllitidis (L.) Sw. & $\mathrm{T}$ & $\mathrm{SF}, \mathrm{DE}$ & NEO & $1865^{\#}$ \\
\hline Anemia tomentosa (Sav.) Sw. & $\mathrm{T}$ & DE, LG & NEO & $1868^{\#}$ \\
\hline Anemia sp. & $\mathrm{T}$ & $\mathrm{DE}$ & - & $1869^{\#}$ \\
\hline \multicolumn{5}{|l|}{ ASPLENIACEAE (8) } \\
\hline Asplenium claussenii Hieron. & $\mathrm{T}, \mathrm{E}$ & SF, GF & NEO & $612^{*}$ \\
\hline Asplenium gastonis Fée & $\mathrm{T}, \mathrm{E}$ & SF, GF & SAM & $575^{*}$ \\
\hline Asplenium inaequilaterale Willd. & $\mathrm{T}$ & $\mathrm{RF}$ & PAN & $1831^{\#}$ \\
\hline Asplenium incurvatum Fée & $\mathrm{T}$ & GF & BRA & $1857^{\#}$ \\
\hline Asplenium jucundum Fée & E & $\mathrm{SF}, \mathrm{GF}$ & NEO & $1811^{\#}$ \\
\hline Asplenium pseudonitidum Raddi & $\mathrm{T}$ & GF & SSE & $1848^{\#}$ \\
\hline Asplenium raddianum Gaudich. & $\mathrm{E}$ & GF & SAM & $1816^{\#}$ \\
\hline Asplenium scandicinum Kaulf. & E & $\mathrm{SF}$ & SAM & $1810^{\#}$ \\
\hline \multicolumn{5}{|l|}{ ATHYRIACEAE (2) } \\
\hline Deparia petersenii (Kunze) M.Kato & $\mathrm{T}$ & $\mathrm{DE}$ & PAN & $614^{*}$ \\
\hline Diplazium cristatum (Desr.) Alston & $\mathrm{T}$ & SF & SAM & $1852^{\#}$ \\
\hline \multicolumn{5}{|l|}{ BLECHNACEAE (9) } \\
\hline Blechnum asplenioides $\mathrm{Sw}$. & $\mathrm{T}$ & LG & SAM & $1828^{\#}$ \\
\hline Blechnum austrobrasilianum de la Sota & $\mathrm{T}$ & $\mathrm{DE}$ & SAM & $599 *$ \\
\hline
\end{tabular}




\begin{tabular}{|c|c|c|c|c|}
\hline FAMILY (nºf species) & Life form & Vegetation & Distribution & Voucher \\
\hline \multicolumn{5}{|l|}{ Species } \\
\hline Blechnum occidentale L. & $\mathrm{T}$ & $\mathrm{DE}$ & NEO & $1827^{\#}$ \\
\hline Blechnum polypodioides Raddi & $\mathrm{T}$ & DE, LG, CF & NEO & $1842^{\#}$ \\
\hline Blechnum sp. & $\mathrm{T}$ & $\mathrm{DE}$ & - & $1854^{\#}$ \\
\hline Lomariocycas schomburgkii (Klotzsch) Gasper \& A.R.Sm. & $\mathrm{T}$ & SW, RS & NEO & $1795^{\#}$ \\
\hline Lomaridium acutum (Desv.) Gasper \& V.A.O.Dittrich & $\mathrm{T}, \mathrm{E}, \mathrm{L}, \mathrm{C}$ & GF, RF & NEO & $569^{*}$ \\
\hline Neoblechnum brasiliense (Desv.) V.A.O. Dittrich & $\mathrm{T}$ & $\mathrm{DE}, \mathrm{SF}$ & NEO & $586^{*}$ \\
\hline Parablechnum cordatum (Desv.) Gasper \& Salino & $\mathrm{T}$ & LG, SW, RS & SAM & $1793^{\#}$ \\
\hline \multicolumn{5}{|l|}{ CYATHEACEAE (6) } \\
\hline Alsophila setosa Kaulf. & A & $\mathrm{SF}, \mathrm{RF}$ & SAM & $1809^{\#}$ \\
\hline Cyathea atrovirens (Langsd. \& Fisch.) Domin & A & DG, SF, SW, RS & SAM & $1790^{\#}$ \\
\hline Cyathea corcovadensis (Raddi) Domin & A & DG, WG, SW & BRA & $1794^{\#}$ \\
\hline Cyathea delgadii Sternb. & A & DG & NEO & $1823^{\#}$ \\
\hline Cyathea phalerata Mart. & A & $\mathrm{SF}, \mathrm{GF}$ & BRA & $1785^{\#}$ \\
\hline Cyathea villosa Willd. & $\mathrm{L}$ & LG & NEO & $1861^{\#}$ \\
\hline \multicolumn{5}{|l|}{ DENNSTAEDTIACEAE (4) } \\
\hline Dennstaedtia globulifera (Poir.) Hieron. & $\mathrm{T}$ & $\mathrm{SF}$ & NEO & $560^{*}$ \\
\hline Dennstaedtia obtusifolia (Willd.) T.Moore & $\mathrm{T}$ & $\mathrm{SF}$ & NEO & $598^{*}$ \\
\hline Hypolepis stolonifera Fée var. stolonifera & $\mathrm{T}$ & $\mathrm{SF}$ & BRA & $573 *$ \\
\hline Pteridium arachnoideum (Kaulf.) Maxon & $\mathrm{T}$ & SF, RS, DE & NEO & $565^{*}$ \\
\hline \multicolumn{5}{|l|}{ DICKSONIACEAE (2) } \\
\hline Dicksonia sellowiana Hook. & A & $\mathrm{RF}$ & NEO & $593 *$ \\
\hline Lophosoria quadripinnata (J.F.Gmel) C. Chr. & $\mathrm{T}$ & $\mathrm{RF}$ & NEO & $1819^{\#}$ \\
\hline \multicolumn{5}{|l|}{ DRYOPTERIDACEAE (13) } \\
\hline Arachniodes denticulata (Sw.) Ching & $\mathrm{T}, \mathrm{L}$ & $\mathrm{RF}$ & NEO & $1824^{\#}$ \\
\hline Ctenitis anniesii (Rosenst.) Copel. & $\mathrm{T}$ & SW & BRA & $1875^{\#}$ \\
\hline Ctenitis distans (Brack) Ching & $\mathrm{T}$ & $\mathrm{SF}$ & BRA & $581 *$ \\
\hline Ctenitis submarginalis (Langsd. \& Fisch.) Ching & $\mathrm{T}$ & $\mathrm{SF}$ & NEO & $608^{*}$ \\
\hline Elaphoglossum burchellii (Baker) C.Chr. & $\mathrm{T}$ & $\mathrm{RF}, \mathrm{GF}, \mathrm{RS}$ & NEO & $1843^{\#}$ \\
\hline Elaphoglossum lingua (C.Presl) Brack. & $\mathrm{E}$ & GF & BRA & $1815^{\#}$ \\
\hline Elaphoglossum macrophyllum (Mett. ex Kuhn) Christ & $\mathrm{T}, \mathrm{L}$ & GF & BRA & $1838^{\#}$ \\
\hline Elaphoglossum pachydermum (Fée) T.Moore & $\mathrm{T}, \mathrm{L}$ & $\mathrm{RF}, \mathrm{GF}$ & BRA & $1805^{\#}$ \\
\hline Elaphoglossum paulistanum Rosenst. & $\mathrm{T}, \mathrm{L}$ & GF & BRA & $1787^{\#}$ \\
\hline Lastreopsis amplissima (C.Presl) Tindale & $\mathrm{T}, \mathrm{L}$ & GF & SAM & $1813^{\#}$ \\
\hline Megalastrum connexum (Kaulf.) A.R.Sm. \& R.C.Moran & $\mathrm{T}$ & $\mathrm{SF}, \mathrm{GF}$ & SAM & $585^{*}$ \\
\hline Polystichum platylepis Fée & $\mathrm{T}$ & $\mathrm{SF}, \mathrm{GF}$ & SSE & $611^{*}$ \\
\hline Ruhmora adiantiformis (G.Forst.) Ching & $\mathrm{T}, \mathrm{E}$ & SW & PAN & $1796^{\#}$ \\
\hline \multicolumn{5}{|l|}{ GLEICHENIACEAE (4) } \\
\hline Dicranopteris flexuosa (Schrad.) Underw. & $\mathrm{T}, \mathrm{L}$ & DG, RS, DE & NEO & $557^{*}$ \\
\hline
\end{tabular}




\begin{tabular}{|c|c|c|c|c|}
\hline FAMILY ( $n^{0}$ of species) & Life form & Vegetation & Distribution & Voucher \\
\hline \multicolumn{5}{|l|}{ Species } \\
\hline Dicranopteris nervosa (Kaulf.) Maxon & $\mathrm{T}, \mathrm{L}$ & DG, DE & SAM & $1820^{\#}$ \\
\hline Sticherus lanuginosus (Fée) Nakai & $\mathrm{T}, \mathrm{L}$ & DG, DE & NEO & $595 *$ \\
\hline Sticherus pruinosus (Mart.) Ching & $\mathrm{T}$ & DG, DE & SAM & $564 *$ \\
\hline \multicolumn{5}{|l|}{ HYMENOPHYLLACEAE (12) } \\
\hline Abrodictyum rigidum (Sw.) Ebihara \& Dubuisson & $\mathrm{L}$ & GF & NEO & $1807^{\#}$ \\
\hline Crepidomanes pyxidiferum (L.) Dubuisson \& Ebihara & $\mathrm{E}$ & SF, GF & PAN & $604 *$ \\
\hline Didymoglossum hymenoides (Hedw.) Desv. & $\mathrm{E}$ & $\mathrm{SF}$ & NEO & $923 !$ \\
\hline Hymenophyllum elegans Spreng. & $\mathrm{L}$ & GF & NEO & $1856^{\#}$ \\
\hline Hymenophyllum fragile (Hedw.) C.V.Morton & $\mathrm{L}$ & GF & NEO & $1801^{\#}$ \\
\hline Hymenophyllum hirsutum (L.) Sw. & $\mathrm{L}$ & GF & NEO & $1844^{\#}$ \\
\hline Hymenophyllum polyanthos (Sw.) Sw. & $\mathrm{E}$ & $\mathrm{RF}$ & PAN & $1798^{\#}$ \\
\hline Hymenophyllum pulchellum Schltdl. \& Cham. & $\mathrm{L}$ & GF & NEO & $1804^{\#}$ \\
\hline Hymenophyllum rufum Fée & $\mathrm{L}$ & GF & SSE & $1803^{\#}$ \\
\hline Polyphlebium angustatum (Carmich.) Ebihara \& Dubuisson & $\mathrm{E}$ & SF, GF & NEO & $1808^{\#}$ \\
\hline Trichomanes anadromum Rosenst. & $\mathrm{E}$ & GF & NEO & $1784^{\#}$ \\
\hline Trichomanes pilosum Raddi & $\mathrm{L}$ & GF, LG & SAM & $1847^{\#}$ \\
\hline \multicolumn{5}{|l|}{ LINDSAEACEAE (1) } \\
\hline Lindsaea botrychioides A.St.-Hil. & $\mathrm{T}$ & GF & BRA & $1840^{\#}$ \\
\hline \multicolumn{5}{|l|}{ LOMARIOPSIDACEAE (1) } \\
\hline Nephrolepis cordifolia (L.) C.Presl & $\mathrm{E}$ & $\mathrm{RF}$ & PAN & $1841^{\#}$ \\
\hline \multicolumn{5}{|l|}{ LYCOPODIACEAE (6) } \\
\hline Lycopodium clavatum $\mathrm{L}$. & $\mathrm{T}$ & WG & PAN & $1822^{\#}$ \\
\hline Palhinhaea cernua (L.) Franco \& Vasc. & $\mathrm{T}$ & WG & PAN & $576^{*}$ \\
\hline Phlegmariurus flexibilis (Fée) B.Øllg. & $\mathrm{E}$ & GF & BRA & $1814^{\#}$ \\
\hline Phlegmariurus heterocarpon (Fée) B.Øllg. & $\mathrm{E}$ & $\mathrm{RF}$ & SAM & $1855^{\#}$ \\
\hline Phlegmariurus reflexus (Lam.) B.Øllg. & $\mathrm{T}$ & $\mathrm{DE}$ & NEO & $570^{*}$ \\
\hline Pseudolycopodiella meridionallis (Underw. \& Lloyd) Holub & $\mathrm{T}, \mathrm{L}$ & WG & NEO & $1821^{\#}$ \\
\hline \multicolumn{5}{|l|}{ POLYPODIACEAE (19) } \\
\hline Campyloneurum angustifolium (Sw.) Fée & $\mathrm{E}$ & $\mathrm{SF}, \mathrm{RF}$ & NEO & $603 *$ \\
\hline Campyloneurum nitidum (Kaulf.) C.Presl & $\mathrm{T}, \mathrm{E}, \mathrm{L}$ & $\mathrm{SF}, \mathrm{RF}$ & SAM & $613^{*}$ \\
\hline Cochlidium serrulatum (Sw.) L.E.Bishop & $\mathrm{L}$ & LG & PAN & $1829^{\#}$ \\
\hline Microgramma squamulosa (Kaulf.) de la Sota & $\mathrm{T}, \mathrm{E}$ & SF, RF, DE & SAM & $556^{*}$ \\
\hline Microgramma vacciniifolia (Langsd. \& Fisch.) Copel. & $\mathrm{E}$ & $\mathrm{RF}$ & NEO & $1825^{\#}$ \\
\hline Pecluma paradiseae (Langsd. \& Fisch.) M.G.Price & $\mathrm{T}, \mathrm{E}$ & $\mathrm{RF}$ & BRA & $1789^{\#}$ \\
\hline Pecluma pectinatiformis (Lindm.) M.G.Price & $\mathrm{E}$ & SF, RF, GF & SAM & $1817^{\#}$ \\
\hline Pecluma recurvata (Kaulf.) M.G.Price & $\mathrm{E}$ & SF, GF & BRA & $567 *$ \\
\hline Pecluma sicca (Lindm.) M.G.Price & $\mathrm{E}$ & SF, RF & SAM & $602 *$ \\
\hline Pecluma singeri (de la Sota) M.G.Price & $\mathrm{T}, \mathrm{H}$ & $\mathrm{SF}$ & SAM & $1818^{\#}$ \\
\hline
\end{tabular}




\begin{tabular}{|c|c|c|c|c|}
\hline FAMILY ( $n^{0}$ of species) & Life form & Vegetation & Distribution & Voucher \\
\hline \multicolumn{5}{|l|}{ Species } \\
\hline Pecluma truncorum (Lindm.) M.G.Price & $\mathrm{E}$ & SF, GF & BRA & $1812^{\#}$ \\
\hline Phlebodium pseudoaureum (Cav.) Lellinger & $\mathrm{E}$ & SF & NEO & $922 !$ \\
\hline Pleopeltis hirsutissima (Raddi) de la Sota & $\mathrm{T}, \mathrm{E}, \mathrm{L}$ & SF, RF, DE & SAM & $611 *$ \\
\hline Pleopeltis macrocarpa (Bory ex Willd.) Kaulf. & $\mathrm{E}$ & $\mathrm{RF}$ & NEO & $1834^{\#}$ \\
\hline Pleopeltis minima (Bory) J.Prado \& R.Y.Hirai & $\mathrm{E}$ & $\mathrm{SF}$ & SAM & $1853^{\#}$ \\
\hline Pleopeltis pleopeltifolia (Raddi) Alston & $\mathrm{E}$ & $\mathrm{SF}, \mathrm{RF}$ & SAM & $555^{*}$ \\
\hline Serpocaulon catharinae (Langsd. \& Fisch.) A.R.Sm. & E, L & $\mathrm{SF}, \mathrm{RF}$ & BRA & $558^{*}$ \\
\hline Serpocaulon latipes (Langsd. \& Fisch.) A.R.Sm. & $\mathrm{T}$ & $\mathrm{SF}, \mathrm{RF}$ & BRA & $1873^{\#}$ \\
\hline Serpocaulon vacillans (Link) A.R.Sm. & $\mathrm{T}$ & SF, SW & SAM & $1872^{\#}$ \\
\hline \multicolumn{5}{|l|}{ PTERIDACEAE (18) } \\
\hline Adiantopsis chlorophylla (Sw.) Fée & $\mathrm{T}$ & DG, WG, DE & NEO & $578^{*}$ \\
\hline Adiantopsis regularis Moore & $\mathrm{T}$ & GF & SAM & $1799^{\#}$ \\
\hline Adiantum pseudotinctum Hieron. & $\mathrm{T}$ & SF & SAM & $566^{*}$ \\
\hline Adiantum raddianum C.Presl & $\mathrm{T}$ & $\mathrm{SF}, \mathrm{RF}$ & NEO & $592 *$ \\
\hline Doryopteris crenulans (Desv.) Link & $\mathrm{T}$ & DG, DE & SAM & $574 *$ \\
\hline Doryopteris concolor (Langsd. \& Fisch.) Kuhn & $\mathrm{T}, \mathrm{L}$ & SF & PAN & $562 *$ \\
\hline Doryopteris lomariacea Klotzsch & $\mathrm{T}$ & WG & SAM & $1800^{\#}$ \\
\hline Doryopteris majestosa Yesilyurt & $\mathrm{T}$ & SF & SAM & $564 *$ \\
\hline Doryopteris ornithopus (Hook. \& Baker) J.Sm. & $\mathrm{L}$ & LG & BRA & $1832^{\#}$ \\
\hline Doryopteris pentagona Pic.Serm. & $\mathrm{T}, \mathrm{E}$ & $\mathrm{SF}, \mathrm{RF}$ & SAM & $561 *$ \\
\hline Pityrogramma calomelanos (L.) Link & $\mathrm{T}$ & $\mathrm{DE}$ & NEO & $1835^{\#}$ \\
\hline Pityrogramma chaerophylla (Desv.) Domin & $\mathrm{T}$ & SF & NEO & $589 *$ \\
\hline Pityrogramma trifoliata (L.) R.M.Tryon & $\mathrm{T}$ & SW & NEO & $1833^{\#}$ \\
\hline Pteris deflexa Link & $\mathrm{T}$ & $\mathrm{SF}$ & NEO & $582 *$ \\
\hline Pteris lechleri Mett. & $\mathrm{T}$ & SF & SAM & $588^{*}$ \\
\hline Pteris vittata $\mathrm{L}$. & $\mathrm{T}$ & $\mathrm{DE}$ & PAN & $1830^{\#}$ \\
\hline $\begin{array}{l}\text { Tryonia areniticola (Schwartsb. \& Labiak) Schuettp., } \\
\text { J.Prado \& A.T.Cochran }\end{array}$ & $\mathrm{L}$ & LG, GF & BRA & $1802^{\#}$ \\
\hline Vittaria lineata (L.) Sm. & $\mathrm{E}$ & $\mathrm{SF}, \mathrm{RF}$ & NEO & $1836^{\#}$ \\
\hline \multicolumn{5}{|l|}{ SALVINIACEAE (2) } \\
\hline Azolla filiculoides Lam. & Q & $\mathrm{RE}$ & PAN & $1018 !$ \\
\hline Salvinia auriculata Aubl. & Q & $\mathrm{RE}$ & NEO & $1851^{\#}$ \\
\hline \multicolumn{5}{|l|}{ SELAGINELLACEAE (4) } \\
\hline Selaginella decomposita Spring & $\mathrm{L}$ & LG & BRA & $1791^{\#}$ \\
\hline Selaginella flexuosa Spring & $\mathrm{L}$ & LG, GF & NEO & $1792^{\#}$ \\
\hline Selaginella marginata (Humb. \& Bonpl. ex Willd.) Spring & $\mathrm{T}$ & GF, SW, RS & NEO & $577 *$ \\
\hline Selaginella sulcata (Desv. ex Poir.) Spring & $\mathrm{T}$ & SF, WG & SAM & $587 *$ \\
\hline \multicolumn{5}{|l|}{ THELYPTERIDACEAE (15) } \\
\hline Amauropelta amambayensis (Ponce) Salino \& T.E.Almeida & $\mathrm{T}$ & $\mathrm{SF}, \mathrm{DE}$ & SSE & $609^{*}$ \\
\hline Amauropelta araucariensis (Ponce) Salino \& T.E.Almeida & $\mathrm{T}$ & $\mathrm{SF}$ & SSE & $580 *$ \\
\hline
\end{tabular}




\begin{tabular}{|c|c|c|c|c|}
\hline $\begin{array}{l}\text { FAMILY (no of species) } \\
\text { Species }\end{array}$ & Life form & Vegetation & Distribution & Voucher \\
\hline Amauropelta decurtata (Link) Salino \& T.E.Almeida & $\mathrm{T}$ & $\mathrm{DE}$ & SAM & $1862^{\#}$ \\
\hline Amauropelta opposita (Vahl) Pic.Serm. & $\mathrm{T}$ & $\mathrm{DE}$ & NEO & $1850^{\#}$ \\
\hline $\begin{array}{l}\text { Amauropelta pachyrhachis (Kunze ex Mett.) Salino \& } \\
\text { T.E.Almeida }\end{array}$ & $\mathrm{T}$ & $\mathrm{DE}$ & NEO & $1849^{\#}$ \\
\hline Amauropelta raddii (Rosenst.) Salino \& T.E.Almeida & $\mathrm{L}$ & GF & SSE & $1788^{\#}$ \\
\hline Amauropelta rivularioides (Fée) Salino \& T.E.Almeida & $\mathrm{T}$ & DG, WG, SW, RS, DE & SAM & $597 *$ \\
\hline Amauropelta sp. & $\mathrm{T}$ & $\mathrm{DE}$ & - & $1782^{\#}$ \\
\hline Christella conspersa (Schrad.) Á.Löve \& D.Löve & $\mathrm{T}$ & $\mathrm{DE}$ & NEO & $1871^{\#}$ \\
\hline Christella dentata (Forssk.) Brownsey \& Jermy & $\mathrm{T}$ & $\mathrm{DE}$ & PAN & $607 *$ \\
\hline Christella hispidula (Decne.) Holttum & $\mathrm{T}$ & SF & PAN & $572 *$ \\
\hline Cyclossorus interruptus (Willd.) H.Ito & $\mathrm{T}$ & SW & PAN & $1797^{\#}$ \\
\hline Goniopteris burkartii C.Chr. ex Abbiatti & $\mathrm{T}$ & $\mathrm{RF}$ & SAM & $600^{*}$ \\
\hline Goniopteris riograndensis (Lindm.) Ching & $\mathrm{T}$ & $\mathrm{RF}$ & SAM & $605^{*}$ \\
\hline Macrothelypteris torresiana (Gaudich.) Ching & $\mathrm{T}$ & $\mathrm{DE}$ & PAN & $583 *$ \\
\hline
\end{tabular}

Regarding life forms (Tab. 1), 63 species $(48.9 \%)$ are terrestrial herbs, 23 epiphytes $(17.9 \%), 14$ lithophytes (10.9\%), six terrestrial arborescents $(4.6 \%)$, two aquatics $(1.5 \%)$ and 21 generalists $(16.3 \%)$. Considering accidental, facultative and mandatory epiphytes, 33 species were recorded $(25.5 \%)$. Pecluma singeri (de la Sota) M.G.Price was the only species growing as hemiepiphyte, however it is also considered a terrestrial herb.

In regard to the vegetation type (Tab. 1), 79 species $(61.2 \%)$ were growing in woody forests, $32(24.8 \%)$ in herbaceous-shrubby environments, $16(12.4 \%)$ in both vegetation formation and two $(1.5 \%)$ in aquatic vegetation. The richest phytophysiognomies with non-overlapping species were the secondary forests with 19 species $(14.7 \%)$, followed by gallery forests (18 spp. $13.9 \%$ ), human disturbed environments (15 spp. - 11.6\%), riparian forests (12 spp. - 9.3), litholic grassland (5 spp. - 3.8\%), swamp areas and wet grassland (4 spp. - $3.1 \%$ each), alagados reservoir (2 spp. - 1.5\%) and dry grassland with one species $(0.7 \%)$. Riparian slabstone environment was the only phytophysiognomy without exclusive species. One the other hand, 49 species $(37.9 \%)$ were found in two or more phytophysiognomies, highlighting that the vast majority of species of ferns and lycophytes in "Campos Gerais" are not restricted to one type of environment.
Concerning the geographical distribution of the species (Tab. 1), most of them were Neotropical (48 species - 37.3\%), followed by South American species (36 spp. - 27.9\%), endemic to Brazil (20 spp. - 15.5\%), Pantropical (16 spp. - 12.4\%) and 6 $(4.6 \%)$ are endemic to southern/southeastern Brazil.

\section{Discussion}

It was found in Carambeí $29 \%$ of the total species cited for the Paraná state, which presents 444 species of ferns and lycophytes (Labiak 2014b). The number of species is consistent with other studies conducted in the "Campos Gerais" region (Schwartsburd and Labiak 2007; Michelon \& Labiak 2013), as well as the most representative families and genera (Tab. 2). The richest fern families found in Carambeí agree with several studies performed in the Atlantic Rain Forest, which constantly cite the following as the most diverse families, regardless of the order, Polypodiaceae, Thelypteridaceae, Pteridaceae, Dryopteridaceae, Aspleniaceae, Blechnaceae and Hymenophyllaceae (see Salino 1996; Salino \& Joly 2001; Mynssen \& Windisch 2004; Salino et al. 2005; Nóbrega \& Prado 2008; Gasper \& Savegnani 2010; Matos et al. 2010; Lautert et al. 2015; Mazziero \& Nonato 2015; Mazziero et al. 2015). It is also true for other Brazilian biomes; Prado et al. (2015) suggest that these families are the most diverse in Brazil and as well as Tryon \& Tryon (1982) in the Neotropics. 
Table 2 - Comparison between ferns and lycophytes floras in the "Campos Gerais" region. The comparison areas are represented by Guartelá = Guartelá State Park (Michelon and Labiak 2013), Vila Velha = Vila Velha State Park (Schwartsburd and Labiak 2007), Klabin = Klabin Ecological Park (Sakagami 2006). Families = the three richest families, Genera = the most representative genera, Epiphytes = number of species, Endemism $=$ percentage of species endemic to Brazil.

\begin{tabular}{|c|c|c|c|c|c|}
\hline & Richness & Families & Genera & Epiphytes & Endemism \\
\hline This study & 129 & $\begin{array}{l}\text { Polypodiaceae } \\
\text { Pteridaceae } \\
\text { Thelypteridaceae }\end{array}$ & $\begin{array}{l}\text { Asplenium } \\
\text { Amauropelta } \\
\text { Doryopteris } \\
\text { Hymenophyllum } \\
\text { Pecluma }\end{array}$ & 33 & $20 \%$ \\
\hline Guartelá & 164 & $\begin{array}{l}\text { Polypodiaceae } \\
\text { Pteridaceae } \\
\text { Dryopteridaceae }\end{array}$ & $\begin{array}{l}\text { Asplenium Amauropelta } \\
\text { Phlegmariurus } \\
\text { Pecluma } \\
\text { Elaphoglossum }\end{array}$ & 54 & $25 \%$ \\
\hline Vila Velha & 152 & $\begin{array}{l}\text { Polypodiaceae } \\
\text { Pteridaceae } \\
\text { Dryopteridaceae }\end{array}$ & $\begin{array}{l}\text { Asplenium } \\
\text { Pecluma } \\
\text { Amauropelta }\end{array}$ & 30 & $18 \%$ \\
\hline Klabin & 121 & $\begin{array}{l}\text { Pteridaceae Polypodiaceae } \\
\text { Blechnaceae }\end{array}$ & $\begin{array}{l}\text { Asplenium } \\
\text { Blechnum } \\
\text { Amauropelta } \\
\text { Doryopteris Pecluma }\end{array}$ & 24 & $13 \%$ \\
\hline
\end{tabular}

Among the three families of lycophytes cited for Brazil (Prado et al. 2015), we recorded two in Carambeí (Lycopodiaceae and Selaginellaceae). These families are the most diverse in Brazil (Prado et al. 2015) and are frequently cited as the richest in several studies (e.g., Salino \& Almeida 2008; Mazziero et al. 2015).

Among the genera, Asplenium and Amauropelta were the richest followed by Doryopteris, Hymenophyllum and Pecluma, all of them have shown an expressive richness in the "Campos Gerais" (Tab. 2) (Schwartsburd \& Labiak 2007; Michelon \& Labiak 2013). The combination of those genera as the most expressive shows a tendency for the ferns' diversity in Paraná's Second Plateau vegetation (Tab. 2). In the Atlantic rain forest, Asplenium and Amauropelta usually figure as the richest genera, however this is not true for Doryopteris, Hymenophyllum and Pecluma. Blechnum, as traditionally treated, also appears as one of the most relevant genus in "Campos Gerais", however, a recent taxonomic revision suggest their segregation in several genera (Gasper et al. 2016). Thus, now at least six genera are found in "Campos Gerais": Austroblechnum, Blechnum, Lomariocycas, Lomaridium, Neoblechnum and Parablechnum. The same way Thelypteris, now, is divided in at least five genera in "Campos Gerais":
Amauropelta, Christella, Cyclossorus, Goniopteris and Steiropteris (Almeida et al. 2015; Salino et al. 2015).

Two possible hybrids were found in study area. The first one belongs to genus Anemia (Anemia sp. in Tab. 1) and the second belongs to genus Blechnum (Blechnum sp. in Tab. 1). In the first case, the specimen was found growing in a slope among Anemia tomentosa (Sav.) Sw. (subgenus Captophyllum) and Anemia phyllitidis (L.) Sw. (subgenus Anemia) and presents morphological characters of both possible parental species. It's probably product of hybridization, a common process in the genus Anemia, but rare between subgenera (Mickel 1962, 1982). The second case of hybridizations is Blechnum sp. which presents intermediate characters between the monomorphic species of Blechnum found in this study, such as $B$. austrobrasilianum and $B$. polypodioides. Hybridization is also common in Blechnum (Moran 1995b), and well documented in the "Campos Gerais" region (Michelon and Labiak 2013).

Terrestrial and epiphyte species are the most diverse guilds of life forms in ferns and lycophytes in Brazil (Prado et al. 2015), which agrees with our result for Carambeí. These guilds of life forms are cited in studies through Brazil independently of 
the biome (Mynssen \& Windisch 2004; Salino et al. 2005; Xavier \& Barros 2005; Nóbrega \& Prado 2008; Zuquim et al. 2008; Gasper \& Sevegnani 2010; Matos et al. 2010; Fernandes et al. 2012; Souza et al. 2012; Salino et al. 2013; Mazziero \& Nonato 2015; Mazziero et al. 2015) the same way in Carambeí and other studies carried out in the inlands of Paraná (Tab. 2) (Schwartsburd \& Labiak 2007; Michelon \& Labiak 2013; Lautert et al. 2015). Epiphytes were the second guild in number of species in Carambei and in the other inland regions of Paraná (Tab. 2) (Schwartsburd \& Labiak 2007; Michelon \& Labiak 2013; Lautert et al. 2015) as well in other areas of Atlantic Rain Forest domain (Salino 1996; Salino \& Joly 2001; Mynssen \& Windisch 2004; Nóbrega \& Prado 2008; Gasper \& Savegnani 2010; Matos et al. 2010; Mazziero \& Nonato 2015; Mazziero et al. 2015; Lautert et al. 2015).

The preponderance of woody forests as the preferential vegetation type for ferns and lycophytes is in agreement with Prado et al. (2015) that suggest the Ombrophilous Forests are the most diverse vegetation type, especially in the Atlantic Rain Forest domain, which is corroborated by several studies in these areas, for example Salino \& Almeida (2008), Matos et al. (2010), Souza et al. (2012) and Mazziero et al. (2015). Secondary forests areas are the most relevant phytophysiognomies by harboring a high richness of exclusive and overlapping species. This could be related to the well preserved state of these areas and also to the fact that secondary forests provide great diversity of niches for these species. Moreover, human disturbed environments also showed high richness of exclusive and overlapping species and it is probably related to the species's preference to open and disturbed areas, for instance: Amauropelta, Dicranopteris, Pteridium and Sticherus (Salino \& Semir 2002; 2004; Melthreter et al. 2010). An interesting fact of Carambeí ferns and lycophytes flora is the low number of species in swamp areas (Tab. 1). Generally these areas shelter a high number of species compared with other phytophysiognomies in the inlands as seen in some studies in São Paulo state by Salino (1996), Salino \& Joly (2001), Nóbrega \& Prado (2008) and Mazziero \& Nonato (2015).

Species with wide geographical distribution are generally well represented in Brazilian surveys (Athayde-Filho et al. 2003; Costa \& Pietrobom 2007, 2010; Melo \& Salino 2007; Salino \& Almeida 2008; Zuquim et al. 2008; Gasper \&
Savegnani 2010; Matos et al. 2010; Fernandes et al. 2012; Macedo et al. 2013; Mazziero et al. 2015) as observed in this study and in other areas of inland of Paraná (Tab. 2) (Schwartsburd \& Labiak 2007; Michelon \& Labiak 2013). We found four exotics and sub-spontaneous species in Brazil (Deparia petersenii, Macrothelypteris torresiana, Pteris vittata and Christella dentata). They have a broad geographic distribution (Prado et al. 2015) and generally occur in disturbed places in the studied areas (Salino \& Almeida 2008; Mazziero et al. 2015). In the other hand, some species could be interpreted as rare in this region of Paraná, it is the case of Arachniodes denticulata, Cyathea villosa, Phlegmariurus flexibilis and Phlegmariurus heterocarpon, all with few records in "Campos Gerais". Tryonia areniticola is endemic to the sandstones outcrops in Paraná and São Paulo states (Schwartsburd \& Labiak 2008) and Dicksonia sellowiana is an endangered species in Brazil (Santiago et al. 2013).

Our study shows the importance of preserving the remnants of vegetation in Carambeí municipality, included in the "Campos Gerais", even for the small ones, given the relevant number of species as well as by the presence of endangered and rare species of ferns and lycophytes.

\section{Acknowledgments}

The authors are grateful to reviewers and $\mathrm{PhD}$. Fabiana R. Nonato for help in reviewing the English. We also thank PhD. Lana S. Sylvestre by the determination of Asplenium jucundum.

\section{References}

Almeida TE, Hennequin S, Schneider H, Smith AR, Batista JAN, Ramalho AJ, Proite K \& Salino A (2015) Towards a phylogenetic generic classification of Thelypteridaceae: Additional sampling suggests alteration of neotropical taxa and further study of paleotropical genera. Molecular Phylogenetics and Evolution 94: 688-700.

Athayde Filho FP, Pereira VS, Smidt EC \& Nonato FR (2003) Pteridófitas do Parque Estadual da Ilha Anchieta (PEIA). Bradea 9: 55-66.

Christenhusz MJM, Schwartsburd PB \& Labiak PH (2009) Hymenophyllum filmenofilicum (Hymenophyllaceae: Pteridophyta): a new epipetric filmy fern from Paraná, southern Brazil. Kew Bulletin 64: 175-178.

Cordeiro Santos LJ, Oka-Fiori C, Canali NE, Fiori AP, Da Silveira CT \& Franca Da Silva JM (2009) Morphostructural mapping of Parana state, Brazil. Journal of Maps 5: 170-178. 
Costa FRC (2004) Structure and composition of the ground-herb community in a terra-firme Central Amazonian forest. Acta Amazonica 34: 53-59.

Costa JM \& Pietrobom MR (2007) Pteridófitas (Lycophyta e Monylophyta) da Ilha de Mosqueiro, município de Belém, estado do Pará, Brasil. Boletim do Museu Paraense Emílio Goeldi 2: 45-55.

Costa JM \& Pietrobom MR (2010) Samambaias e licófitas do Parque Ecológico de Gunma, município de Santa Bárbara do Pará, estado do Pará, Brasil. Rodriguésia 61: 223-232.

Fernandes RS, Maciel S \& Pietrobom MR (2012) Licófitas e monilófitas das Unidades de Conservação da Usina Hidrelétrica - UHE de Tucuruí, Pará, Brasil. Hoehnea 39: 247-285.

Fidalgo O \& Bononi VLR (1984) Técnicas de coleta, preservação e herborização de material botânico. Manual do Instituto de Botânica no 4, São Paulo. 62p.

Fundação SOS Mata Atlântica \& INPE - Instituto Nacional de Pesquisas Espaciais (2001) Atlas dos remanescentes florestais da Mata Atlântica e ecossistemas associados no período de 1995-2000. Fundação SOS Mata Atlântica e INPE, São Paulo. 61p.

Gasper AL \& Sevegnani L (2010) Lycophyta e samambaias do Parque Nacional da Serra do Itajaí, Vale do Itajaí, SC, Brasil. Hoehenea 37: 755-767.

Gasper AL, Dittrich VAO, Smith AR \& Salino A (2016) A classification for Blechnaceae (Polypodiales: Polypodiopsida): new genera, resurrected names, and combinations. Phytotaxa 275: 191-227.

Gentry AH (1990) Floristic similarities and differences between Southern Central America and upper and Central Amazonia. In: Gentry AH (ed.) Four neotropical rain forests. Yale University Press, New Haven. Pp. 141-160.

IPNI - The International Plant Names Index (2012) The International Plant Names Index. Available at $<$ http://www.ipni.org > . Access on 6 April 2017.

Labiak PH (2014a) Aspectos fitogeográficos do Paraná. In: Kaehler M, Goldenberg R, Labiak PH, Ribas OS, Vieira AOS \& Hatschbach GG (eds.) Plantas vasculares do Paraná. Ed. UFPR, Curitiba. Pp. 7-22.

Labiak PH (2014b) Samambaias e licófitas. In: Kaehler M, Goldenberg R, Labiak PH, Ribas OS, Vieira AOS \& Hatschbach GG (eds.) Plantas vasculares do Paraná. Ed. UFPR, Curitiba. Pp. 45-55.

Lautert M, Temponi LG, Viveros RS \& Salino A (2015) Lycophytes and ferns composition of Atlantic Forest conservation units in western Paraná with comparisons with other areas in southern Brazil. Acta Botanica Brasilica 29: 499-508.

Maack R (1981) Geografia física do estado do Paraná. José Olympio, Rio de Janeiro. 450p.

Macedo TS, Góes Neto A \& Nonato FR (2013) Análise florística e fitogeografia das samambaias e licófitas de um fragmento de Mata Atlântica na Serra da
Jibóia, Santa Terezinha, Bahia, Brasil. Rodriguésia 64: 561-572.

Matos FB, Amorim AM \& Labiak PH (2010) The ferns and lycophytes of a montane tropical forest in southern Bahia, Brazil. Journal of the Botanical Research Institute of Texas 4: 333-346.

Mazziero FFF, Labiak PH \& Paciencia M (2015) Checklist of ferns and lycophytes from the Parque Estadual Turístico do Alto Ribeira, Iporanga, São Paulo, Brazil. Check List 11: 1791.

Mazziero FFF \& Nonato FR (2015) Ferns and lycophytes from Jaú, São Paulo, Brazil. Check List 11: 1798.

Melo LCN \& Salino A (2007) Pteridófitas em fragmentos florestais na APA Fernão Dias, Minas Gerais, Brasil. Rodriguésia 58: 207-220.

Melthreter K, Walker LR \& Sharpe JM (2010) Fern ecology. Cambridge University Press, Cambridge. 460p.

Michelon C \& Labiak PH (2013) Samambaias e licófitas do Parque Estadual do Guartelá, PR, Brasil. Hoehnea 40: 191-204.

Mickel JT (1962) A monographic study of the fern genus Anemia, subgenus Captophyllum. Iowa State Journal of Science 4: 349-382.

Mickel JT (1982) The genus Anemia (Schizaeaceae) in Mexico. Brittonia 34: 388-413.

Moran RC (1995a) The importance of mountains to pteridophytes, with emphasis on neotropical rain forests. In: Churchill SP, Balslev H, Forero E \& Luteyn JL (eds.) Biodiversity and conservation on neotropical montane forests. New York Botanical Garden, New York. Pp. 359-563.

Moran RC (1995b) Blechnaceae. In: Davidse G, Sousa M \& Knapp S (eds.) Flora Mesoamericana. Vol. 1. Universidad Nacional Autónoma do México, Ciudad de México. Pp. 325-333.

Moran RC (2008) Diversity, biogeography and floristics. In: Ranker TA \& Haufler CH (eds.) Biology and evolution of ferns and lycophytes. Cambridge University Press, Cambridge. Pp. 367-394.

Mynssen CM \& Windisch PG (2004) Pteridófitas da Reserva Rio das Pedras, Mangaratiba, RJ, Brasil. Rodriguesia 55: 125-156.

Nóbrega GA \& Prado J (2008) Pteridófitas da vegetação nativa do Jardim Botânico Municipal de Bauru, estado de São Paulo, Brasil. Hoehnea 35: 7-55.

Paciencia MLB (2008) Diversidade de pteridófitas em gradientes de altitude na Mata Atlântica do estado do Paraná, Brasil. Tese de Doutorado. Universidade de São Paulo, São Paulo. 229p.

PPG I (2016) A community-derived classification for extant lycophytes and ferns. Journal of Sytematics Evolution 54: 563-603.

Prado J, Sylvestre LS, Labiak PH, Windisch PG, Salino A, Barros ICL, Hirai RY, Almeida TE, Santiago ACP, Kieling-Rubio MA, Pereira AFN, Øllgaard B, Ramos CG, Mickel JT, Dittrich VAO, Mynssen 
CM, Schwartsburd PB, Condack JPS, Pereira JBS \& Matos FB (2015) Diversity of ferns and lycophytes in Brazil. Rodriguésia 66: 1073-1083.

Sakagami CR (2006) Pteridófitas do Parque Ecológico da Klabin, Telêmaco Borba, Paraná, Brasil. Dissertação de Mestrado, Universidade Federal do Paraná, Curitiba. 212p.

Salino A (1996) Levantamento das pteridófitas da Serra do Cuscuzeiro, Analândia, SP, Brasil. Revista Brasileira de Botânica 19: 173-178.

Salino A \& Almeida TE (2008) Pteridófitas do Parque Estadual do Jacupiranga, SP, Brasil. Acta Botanica Brasilica 22: 983-991.

Salino A \& Joly CA (2001) Pteridophytes of three remnants of gallery forests in the Jacaré-Pepira river basin, São Paulo state, Brazil. Boletim do Herbário Ezechias Paulo Heringer 8: 5-15.

Salino A \& Semir J (2002) Thelypteridaceae (Polypodiophyta) do estado de São Paulo: Macrothelypteris e Thelypteris subgêneros Cyclosorus e Steiropteris. Lundiana 3: 9-27.

Salino A \& Semir J (2004) Thelypteris subg. Amauropelta (Kunze) A.R. Sm. (Thelypteridaceae - Pteridophyta) no estado de São Paulo, Brasil. Lundiana 5: 83-112.

Salino A, Almeida TE \& Smith AR (2015) New combinations in Neotropical Thelypteridaceae. Phytokey 57: 11-50.

Salino A, Mota NFO \& Almeida TE (2013) Lycophytes and monilophytes in Rio Preto State Park, Minas Gerais, Brazil. Acta Botanica Brasilica 27: 252-263.

Salino A, Silva SM, Dittrich VAO \& Britez RM (2005)
Flora pteridofítica. In: Marques MCM \& Britez RC (eds.) História natural e conservação da Ilha do Mel. Ed. UFPR, Curitiba. Pp. 85-101.

Santiago ACP, Mynssen CM, Maurenza D, Penedo TSA \& Sfair JC (2013) Dickoniaceae. In: Martinelli G \& Moraes MA (eds.) Livro vermelho da flora do Brasil. Centro Nacional de Conservação da Flora, Rio de Janeiro. Pp. 475-476.

Schwartsburd PB \& Labiak PH (2007) Pteridófitas do Parque Estadual de Vila Velha, Ponta Grossa, Paraná, Brasil. Hoehnea 34: 159-209.

Schwartsburd PB \& Labiak PH (2008) Eriosorus areniticola (Pteridaceae), a new species from Brazil. American Fern Journal 98: 160-163.

Schwartsburd PB, Labiak PH \& Salino A (2007) A new species of Ctenitis (Dryopteridaceae) from southern Brazil. Brittonia 59: 29-32.

Souza FS, Salino A, Viana PL \& Salimena FRG (2012) Pteridófitas da Serra Negra, Minas Gerais, Brasil. Acta Botanica Brasilica 26: 378-390.

Tryon RM \& Tryon AF (1982). Ferns and allied plants, with special reference to tropical America. SpringerVerlag, New York. 857p.

Xavier SRS \& Barros ICL (2005) Pteridoflora e seus aspectos ecológicos ocorrentes no Parque Ecológico João Vasconselos Sobrinho, Caruaru, Pernambuco, Brasil. Acta Botanica Brasilica 19: 775-781.

Zuquim G, Costa FRC, Prado J \& Tuomisto H (2008) Guia de samambaias e licófitas da REBIO Uatumã, Amazônia central. Átemma Design Editorial, Manaus. 316p. 